\title{
Safety profile of end-stage heart failure patients implanted with left ventricular assist devices. Krakow two-year observational all-comers study on left ventricular assist device recipients
}

\author{
Paweł Rubiś ${ }^{1}$, Katarzyna Holcman ${ }^{1}$, Bogusław Kapelak², Sylwia Wiśniowska-Śmiałek ${ }^{1}$, \\ Izabela Górkiewicz-Kot ${ }^{2}$, Michał Kaleta ${ }^{2}$, Rafał Drwiła², Hubert Hymczak ${ }^{2}$, Irena Milaniak ${ }^{2}$, \\ Jolanta Siwińska², Maciej Stąpór ${ }^{2}$, Dorota Sobczyk ${ }^{1}$, Andrzej Gackowski ${ }^{3}$, Robert Musiał ${ }^{4}$, \\ Piotr Podolec ${ }^{1}$, Jadwiga Nessler ${ }^{3}$, Karol Wierzbicki ${ }^{2}$ \\ 'Department of Cardiac and Vascular Diseases, Institute of Cardiology, Jagiellonian University Medical College, \\ John Paul II Hospital, Krakow, Poland \\ 2Department of Cardiovascular Surgery and Transplantology, Institute of Cardiology, Jagiellonian University Medical College, \\ John Paul II Hospital, Krakow, Poland \\ ${ }^{3}$ Department of Coronary Disease and Heart Failure, Institute of Cardiology, Jagiellonian University Medical College, \\ John Paul II Hospital, Krakow, Poland \\ ${ }^{4}$ Department of Anaesthesiology and Intensive Therapy, Jagiellonian University Medical College, John Paul II Hospital, Krakow, Poland
}

\section{INTRODUCTION}

Heart transplantation $(\mathrm{HTx})$ is an established line of treatment for advanced heart failure (HF), but with a shortage of donor organs its impact is limited. Therefore, the demand for efficacious and safe mechanical left ventricular assist devices (LVADs) is increasing. Trials with the latest-generation LVADs have reported survival rates of $85 \%$ to $92 \%$ [1]. According to leading European and American societies, LVADs are an established therapy for end-stage HF [2, 3]. However, LVAD-related complications are still common and include pump thrombosis, HF worsening (HFW), right ventricular failure (RV-HF), drive-line infections (DRI), stroke, and bleeding.

Polish data on LVADs are scarce [4]. Since 1988, more than 500 HTxs have been performed at the John Paul II Hospital in Krakow. In October 2015, an LVAD programme was initiated. The aim of this study is to report up-to-date data on LVADs, including survival rates and complications.

\section{METHODS}

This is a prospective, all-comers study of 32 consecutive patients who underwent LVAD implantation between November 2015 and October 2017. All data collected were based on hospitalisation and outpatient records. The study was approved by the local Ethics Committee.

We created an end-stage HF team, which included cardio-surgeons (B.K. and K.W.), cardiologists, cardio-anaesthetists, and associated personnel. Upon discharge, patients and their families were informed about LVAD management and provided with a 24/7 telephone line.

The primary endpoint was composed of death and pump thrombosis. Secondary (safety) endpoints included: RV-HF, urgent hospitalisation due to HFW, DRI, and bleedings.

\section{Statistical analysis}

Cox proportional hazards models were used to calculate hazard ratios (HRs) and 95\% confidence intervals (Cls) for the primary endpoint, its individual component events, and secondary endpoints. Survival data were analysed using the Kaplan-Meier method and compared with the log-rank test. To examine the associations of selected variables with endpoints of interest (unadjusted analyses and analyses adjusted for age, INTERMACS score, and N-terminal pro-B-type natriuretic peptide [NT-proBNP] level) Cox proportional hazards models were used. All the analyses were conducted in R software, 
Table 1. Comparisons of primary and secondary endpoints in patients stratified according to heart failure (HF) aetiology, INTERMACS score, and time of left ventricular assist device implantation

\begin{tabular}{|c|c|c|c|c|c|c|}
\hline & $\begin{array}{c}\text { DCM } \\
(n=17)\end{array}$ & $\begin{array}{c}\text { ICM } \\
(n=15)\end{array}$ & $\begin{array}{l}\text { INTERMACS } \\
1-2(n=7)\end{array}$ & $\begin{array}{l}\text { INTERMACS } \\
>3(n=25)\end{array}$ & First 16 & Last 16 \\
\hline Primary endpoint & $5(29.4)$ & $3(20)$ & $1(14.3)$ & $7(28)$ & $7(43.7)$ & $1(6.2)$ \\
\hline $\mathrm{HR}, 95 \% \mathrm{Cl}$ & 1 & $0.6(0.12-3.1)$ & 1 & $2.3(0.24-23)$ & $1^{*}$ & $0.086(0.009-0.81)^{*}$ \\
\hline Death & $3(17.6)$ & $1(6.7)$ & $1(14.3)$ & $3(12)$ & $3(18.7)$ & $1(6.2)$ \\
\hline $\mathrm{HR}, 95 \% \mathrm{Cl}$ & 1 & $0.3(0.03-3.6)$ & 1 & $0.82(0.07-9.3)$ & 1 & $0.29(0.03-3.13)$ \\
\hline Pump thrombosis & $2(11.8)$ & $2(13.3)$ & $0(0)$ & $4(16)$ & $4(25)$ & $0(0)$ \\
\hline $\mathrm{HR}, 95 \% \mathrm{Cl}$ & 1 & $1.15(0.14-9.4)$ & 1 & $>1000(<0.01->100)$ & $1^{*}$ & $<0.001(<0.01->100)^{*}$ \\
\hline RV failure & $5(29.4)$ & $4(26.7)$ & $2(28.6)$ & $7(28)$ & $7(43.7)$ & $2(12.5)$ \\
\hline $\mathrm{HR}, 95 \% \mathrm{Cl}$ & 1 & $0.87(0.19-4.1)$ & 1 & $0.97(0.15-6.2)$ & $1^{*}$ & $0.18(0.03-0.95)^{*}$ \\
\hline HF worsening & $6(35.3)$ & $6(40)$ & $1(14.3)$ & $11(44)$ & $9(56)$ & $3(18.7)$ \\
\hline $\mathrm{HR}, 95 \% \mathrm{Cl}$ & 1 & $1.2(0.29-5.1)$ & 1 & $4.7(0.5-45.2)$ & $1 *$ & $0.18(0.04-0.9)^{*}$ \\
\hline Bleedings & $3(17.6)$ & $5(33.3)$ & $1(14.3)$ & $7(28)$ & $5(31.2)$ & $3(18.7)$ \\
\hline $\mathrm{HR}, 95 \% \mathrm{Cl}$ & 1 & $2.3(0.45-12.1)$ & 1 & $2.3(0.24-23.04)$ & 1 & $0.51(0.1-2.62)$ \\
\hline Infections & $5(29.4)$ & $6(40)$ & $3(42.8)$ & $8(32)$ & $5(31.2)$ & $6(37.5)$ \\
\hline $\mathrm{HR}, 95 \% \mathrm{Cl}$ & 1 & $1.6(0.4-6.9)$ & 1 & $0.63(0.1-3.5)$ & 1 & $1.3(0.3-5.7)$ \\
\hline
\end{tabular}

Data are shown as number (percentage); Cl — confidence interval; DCM — dilated cardiomyopathy; HR - hazard ratio; ICM — ischaemic cardiomyopathy; RV — right ventricular; $p$-values for comparison between subgroups: ${ }^{*} p<0.05$

version 3.3.2 (R Foundation for Statistical Computing, Vienna, Austria). All results were considered statistically significant when $p$ was $<0.05$.

\section{RESULTS AND DISCUSSION}

The baseline characteristics of the study population, divided according to HF aetiology into ischaemic $(\mathrm{n}=15)$ and dilated cardiomyopathy (DCM, $\mathrm{n}=17)$, showed that men constituted the majority (94\%) (Suppl. Table 1 - see journal website). New York Heart Association class (ischaemic HF: $4 \pm 0.3$ vs. DCM: $3.8 \pm 0.4 ; p=$ NS) and INTERMACS scores $(2.8 \pm 0.4$ vs. $2.7 \pm 0.7 ; p=N S)$ were similar. The left ventricular (LV) cavity was larger in the DCM group $(79.3 \pm 13.1 \mathrm{~mm}$ vs. $71.7 \pm 6.5 \mathrm{~mm}$; $\mathrm{p}<0.05)$, but the ejection fraction was comparable $(13.3 \% \pm 3.8 \%$ vs. $13.8 \% \pm 3.4 \% ; p=N S)$. NT-proBNP levels were significantly higher in the DCM group $(8960 \pm 10108$ pg/mL vs. $3323 \pm 2294$ pg/mL; $p<0.05)$.

During the observational period of $12.2 \pm 7.3$ months, primary endpoint occurred in eight (24\%) patients; death was reported in four $(12 \%)$, and pump thrombosis in four $(12 \%)$. Endpoints were analysed in all patients and subdivided into three categories: (1) according to HF aetiology: ischaemic vs. non-ischaemic, (2) INTERMACS score 1-2 vs. 3, and (3) first 16 vs. last 16 patients (Table 1). Patients stratified according to HF aetiology or INTERMACS were similar in terms of the of the primary endpoint. However, primary endpoint was more common in the first half compared to the second half of the population (7 [43.7\%] vs. $1[6.2 \%]$; HR 0.086; 95\% Cl 0.009-0.81; $\mathrm{p}<0.05)$. With regard to the primary endpoint components, numbers of deaths were comparable between the groups (3 [18.7\%] vs. 1 [6.2\%]; HR $0.29 ; 95 \% \mathrm{Cl} 0.03-3.13 ; \mathrm{p}=\mathrm{NS}$ ), whereas pump thrombosis was more frequent in the first half compared to the second half of patients $(4[25 \%]$ vs. $0[0 \%] ; p<0.05)$. None of the following variables were predictors of the primary endpoint: LVAD type, prior cardiac arrest, chronic kidney disease (CKD), atrial fibrillation, patent foramen ovale (PFO), or the levels of haemoglobin, creatinine, urea, creatine kinase (CK), CK-MB, D-dimer, troponin, and C-reactive protein.

Right ventricular failure was observed in nine $(27.3 \%)$ patients and was similarly distributed in patients grouped according to HF aetiology and INTERMACS. However, RV-HF was more common in the first 16 LVAD recipients (7 [43.7\%] vs. 2 [12.5\%]; HR 0.18; 95\% Cl 0.03-0.95; $p<0.05)$. PFO was the only independent predictor of RV-HF in a model adjusted for age, INTERMACS score and NT-proBNP level (HR 35.8; 95\% Cl 1.33-969.8; $p<0.05$ ).

Hospitalisation due to HFW occurred in 12 (40\%) patients. HFW was more common in the first half of the population (9 [56\%] vs. 3 [18.7\%]; $p<0.05$ ). Only CKD was an independent predictor of HFW in an adjusted model (HR 1.86; 95\% Cl 1.08-3.96; $\mathrm{p}<0.05)$.

Drive-line infections and bleeding complications occurred in 11 (34\%) and eight (24\%) patients, respectively. The frequencies of both complications were similar in all three patient subgroups.

The LVAD registries report similar baseline characteristics of the device recipients, and the frequency of ischaemic HF 
and DCM is almost 50/50. Recent years have seen a shift towards implanting less seriously ill patients (INTERMACS 3-5) as compared to the historical pattern of LVAD implantations in morbid/dying patients (INTERMACS 1-2) [5].

In the early LVAD trial, REMATCH (1998-2001), one-year survival estimates were $52 \%$ in the LVAD compared to $25 \%$ in the medical group [6]. According to the INTERMACS registry (2008-2014), one- and two-year survival of 12,030 LVAD patients was $80 \%$ and $70 \%$, respectively $[1,5]$. In the recent MOMENTUM 3 (2014-2015) trial 13 (8.5\%) out of 152 HeartMate3 and 14 (9.8\%) out of 142 HeartMatell patients died within six months [7]. Our results are concordant with the published reports. Furthermore, we observed a striking $91.4 \%$ relative risk reduction in the primary endpoint in the 16 patients implanted later.

In the early days, pump thrombosis was reported in almost $20 \%$ of patients but has been reduced to the current incidence of $10 \%$ to $12 \%$. We reported the occurrence of pump thrombosis in four (12\%) patients, which is comparable to the current rates.

Left ventricular assist device supports the systemic ventricle; thus, RV function is crucial for recovery. The frequency of RV-HF after LVAD implantation is reported at 35\% [8]. Surprisingly, we observed, for the first time, that RV-HF was independently associated with the presence of PFO. The mechanisms of this association are unknown. We speculate that during the progression of LV dysfunction, an increase in left atrial pressure could have resulted in the PFO opening, leading to an interatrial shunt. An increase in RV volume may have an additional damaging effect on RV morphology and function.

Apart from the difficulties of continuous anticoagulation with vitamin K antagonists, over time LVAD recipients develop acquired von Willebrand disease. At the same time, many patients develop arteriovenous malformations in the mucosa of the gastrointestinal tract. Taken together, LVAD patients are predisposed to bleedings. In the early REMATCH trail, the frequency of bleeding was $42 \%$ [8]. Clinically relevant bleeding, which is a typical complication, occurred in eight (24\%) patients from our cohort.

Patients with LVADs are particularly predisposed to DRI. The typical site of infection is the exit site of the driveline. DRIs were reported in 17 (12\%) and sepsis in $16(11.4 \%)$ patients out of 140 subjects from the INTERMACS registry [1,5].
In conclusion, a long-term LVAD programme is feasible in the Polish medical system; however, it requires a highly-specialised cardiac centre. The safety profile is comparable to that in experienced Western centres. Over 91\% risk reduction in the latter half of patients supports a learning curve and accumulation of experience. Newly-found associations between PFO and RV-HF need further exploration.

\section{Conflict of interest: none declared}

\section{References}

1. Starling RC, Naka Y, Boyle AJ, et al. Results of the post-U.S. Food and Drug Administration-approval study with a continuous flow left ventricular assist device as a bridge to heart transplantation: a prospective study using the INTERMACS (Interagency Registry for Mechanically Assisted Circulatory Support). J Am Coll Cardiol. 2011; 57(19): 1890-1898, doi: 10.1016/j.jacc.2010.10.062, indexed in Pubmed: 21545946.

2. Ponikowski P, Voors AA, Anker SD, et al. 2016 ESC Guidelines for the diagnosis and treatment of acute and chronic heart failure: The Task Force for the diagnosis and treatment of acute and chronic heart failure of the European Society of Cardiology (ESC). Eur J Heart Fail. 2016; 18(8): 891-975, doi: 10.1016/j. rec.2016.11. 005, indexed in Pubmed: 27894487.

3. Yancy CW, Jessup M, Bozkurt B, et al. 2013 ACCF/AHA guideline for the management of heart failure: a report of the American College of Cardiology Foundation/American Heart Association Task Force on Practice Guidelines. J Am Coll Cardiol. 2013; 62(16): e147-e239, doi: 10.1016/j.jacc.2013.05.019, indexed in Pubmed: 23747642.

4. Litwiński P, Kołsut P, Jasińska M, et al. First implantation of a HeartMate 3 left ventricular assist device in Poland. Kardiol Pol. 2016; 74(9): 1028, doi: 10.5603/KP.2016.0124, indexed in Pubmed: 27654475.

5. Kirklin JK, Naftel DC, Pagani FD, et al. Seventh INTERMACS annual report: 15,000 patients and counting. J Heart Lung Transplant. 2015; 34(12): 1495-1504, doi: 10.1016/j. healun.2015.10.003, indexed in Pubmed: 26520247.

6. Rose EA, Gelijns AC, Moskowitz AJ, et al. Long-term use of a left ventricular assist device for end-stage heart failure. NEngl J Med. 2001;345(20): 1435-1443, doi: 10.1056/NEJMoa012175, indexed in Pubmed: 11794191.

7. Mehra MR, Naka Y, Uriel N, et al. A fully magnetically levitated circulatory pump for advanced heart failure. N Engl J Med. 2017; 376(5): 440-450, doi: 10.1056/NEJMoa1610426, indexed in Pubmed: 27959709.

8. Bellavia D, Iacovoni A, Scardulla C, et al. Prediction of right ventricular failure after ventricular assist device implant: systematic review and meta-analysis of observational studies. Eur J Heart Fail. 2017; 19(7): 926-946, doi: 10.1002/ejhf.733, indexed in Pubmed: 28371221.

Cite this article as: Rubiś P, Holcman K, Kapelak B, et al. Safety profile of end-stage heart failure patients implanted with left ventricular assist devices. Krakow two-year observational all-comers study on left ventricular assist device recipients. Kardiol Pol. 2018; 76(9): 1369-1371, doi: 10.5603/KP.2018.0183. 